\title{
CORRIGENDUM
}

\section{A note on operator semigroups associated to chaotic flows - CORRIGENDUM}

\author{
OLIVER BUTTERLEY \\ doi:10.1112/S0143385714001278, Published by Cambridge University Press, \\ 11 February 2015.
}

In [1], an oversight in the computation of a residue in the formula just before (4.2) led to the omission of the nilpotents. The corrected statement of Theorem 1 is:

THEOREM 1. Suppose that $T_{t}: \mathcal{B} \rightarrow \mathcal{B}$ is a bounded one-parameter semigroup satisfying Assumptions 1, 2, and $3 A$. Then there exists a finite set

$$
\left\{z_{j}\right\}_{j=1}^{N} \subset\{z \in \mathbb{C}:-\lambda<\operatorname{Re}(z) \leq 0,|\operatorname{Im}(z)| \leq \beta\},
$$

a set of finite rank projectors $\left\{\Pi_{j}\right\}_{j=1}^{N}$, a set of nilpotents $\left\{\mathcal{N}_{j}\right\}_{j=1}^{N}$ and an operatorvalued function $t \mapsto P_{t} \in \mathscr{B}(\mathcal{B}, \mathcal{B})$ where $\Pi_{j} P_{t}=P_{t} \Pi_{j}=0, \Pi_{j} \Pi_{k}=\delta_{j k} \Pi_{j}, \Pi_{j} \mathcal{N}_{j}=$ $\mathcal{N}_{j} \Pi_{j}=\mathcal{N}_{j}$ such that

$$
T_{t}=P_{t}+\sum_{j=1}^{N} e^{t z_{j}} e^{t \mathcal{N}_{j}} \Pi_{j} \quad \text { for all } t \geq 0 .
$$

Moreover, for all $\ell<\lambda$ there exists $C_{\ell}>0$ such that, for all $\mu \in \operatorname{Dom}(Z), t \geq 0$,

$$
\left\|P_{t} \mu\right\|_{\mathcal{A}} \leq C_{\ell} e^{-\ell t}\|Z \mu\|_{\mathcal{B}} .
$$

The proof is corrected as follows. In the formula just before (4.2) it is required to calculate $\sum_{j=1}^{N}(1 / 2 \pi i) \int_{\Gamma_{j}} e^{z t} R(z) d z$ where the $\left\{z_{j}\right\}_{j}$ are the poles of $R(z)$ and each $\Gamma_{j}$ is a positive oriented contour containing $z_{j}$ and no other singularity of $R(z)$. Since $R(z)$ is a pseudo-resolvent we represent $R(z)$ by the Laurent expansion in terms of projectors and nilpotents (see [2, equation III-(6.35)] and note that the quasi-nilpotents are actually nilpotents since they are finite rank in this case). Consequently,

$$
\begin{aligned}
\frac{1}{2 \pi i} \int_{\Gamma_{j}} e^{z t} R(z) d z & =\frac{1}{2 \pi i} \int_{\Gamma_{j}} e^{z t}\left[\frac{\Pi_{j}}{z-z_{j}}+\sum_{n=1}^{\infty} \frac{\mathcal{N}_{j}^{n}}{\left(z-z_{j}\right)^{n+1}}\right] d z \\
& =e^{z_{j} t}\left(\Pi_{j}+\sum_{n=1}^{\infty} \frac{t^{n}}{n !} \mathcal{N}_{j}^{n}\right)=e^{t z_{j}} e^{t \mathcal{N}_{j}} \Pi_{j} .
\end{aligned}
$$


The other arguments in the paper hold just as before but now with the additional nilpotent term carried through. The same correction is required to the statement of Theorem 2, that is, (2.5) should read

$$
T_{t}=P_{t}+\sum_{j=1}^{N} e^{t z_{j}} e^{t \mathcal{N}_{j}} \Pi_{j} \quad \text { for all } t \geq 0,
$$

where the $\left\{\Pi_{j}\right\}_{j=1}^{N}$ and $\left\{\mathcal{N}_{j}\right\}_{j=1}^{N}$ are as above. All the other calculations hold as per the original paper.

Acknowledgement. It is a pleasure to thank Carlangelo Liverani for pointing out this inaccuracy.

\section{REFERENCES}

[1] O. Butterley. A note on operator semigroups associated to chaotic flows. Ergod. Th. \& Dynam. Sys., doi:10.1112/S0143385714001278, published online 11 February 2015.

[2] T. Kato. Perturbation Theory for Linear Operators. Springer, Berlin, 1966. 